\title{
PLWC Resources
}

\section{PLWC Expands Information to Help Your Patients Better Manage Side Effects of Cancer and Cancer Treatment}

The Managing Side Effects section of PLWC (www.plwc.org/ sideeffects), adapted from the ASCO Curriculum-Optimizing Cancer Care: The Importance of Symptom Management, helps patients learn about the side effects of cancer and cancer treatment. These easy-to-read articles discuss the definitions, causes, symptoms, and management of 15 of the most common side effects. PLWC recently expanded the Managing Side Effects section to include 20 additional side effects (sidebar: Managing Side Effects: Topics on PLWC). All information has been reviewed by PLWC Editorial Board members Jamie Von Roenn, MD, and Charles Loprinzi, MD.

To help your patients find relevant information about their type of cancer in one place, side effects information that is specific to more than 85 types of cancer will soon be added to the PLWC Guides to Cancer. Visit www.plwc.org/cancer for more information.

Side effect management is also a featured topic each year in PLWC's Ask the ASCO Expert program. On Monday, July 25, 2005, PLWC hosted an Ask the ASCO Expert chat on side effect management with Michael C. Perry, MD, FACP, of the Ellis Fischel Cancer Center at the University of Missouri. Participants asked questions about pain, fatigue, hair loss, depression, anxiety, appetite changes, nausea and vomiting, and sexual side effects. Transcripts from each chat event continue to be a valuable resource for patients and are available at www.plwc/transcripts (sidebar: Ask the ASCO Expert Chat Transcript).

\section{Ask the ASCO Expert Chat Transcript}

The following is excerpted from the Side Effects of Cancer Treatment chat transcript with Jamie Von Roenn, MD, held on Thursday, April 22, 2004.

Good afternoon and welcome. Thank you for joining us. Dr. Von Roenn will now begin taking questions.

Dr. Von Roenn, thank you for taking the time to join us today.

Guest81: How long do chemobrain, memory problems, and thinking difficulties last with chemotherapy? Are these side effects permanent?

Dr. Von Roenn: Somewhere in the neighborhood of $25 \%$ of women who receive adjuvant chemotherapy for breast cancer develop problems with their thinking. Most often, a problem is characterized as difficultly with short-term memory and/or linear thinking. There is inadequate information about this symptom, but it appears that the majority of women recover most, if not all, of their function.

At this point we do not yet know how to predict who is at greatest risk, how to identify co-factors, or prevent the problem. There are currently many clinical trials going on around the country to better understand this symptom as well as to assess potential treatments.

Jim: Can you please talk about radiation treatment's effects on the body, particularly the heart?

Dr. Von Roenn: Radiation therapy causes side effects depending upon where you point the beam. People who have whole brain irradiation lose their hair and frequently develop problems with short-term memory. Radiation therapy to the heart may accelerate the development of coronary artery disease. Radiation to the pelvis may cause long-lasting diarrhea and/or cystitis.

\author{
Managing Side Effects: Topics on PLWC \\ Anemia \\ Anxiety \\ Appetite loss \\ Blocked intestine (GI obstruction) \\ Cognitive problems \\ Constipation \\ Depression \\ Diarrhea \\ Difficulty chewing \\ Difficulty swallowing (dysphagia) \\ Dry mouth (xerostomia) \\ Fluid in the abdomen (ascites) \\ Fluid in the arms or legs (lymphedema) \\ Fluid in the lungs (malignant pleural effusion) \\ Hair loss (alopecia) \\ Hormone deprivation symptoms: Men \\ Hypercalcemia \\ Infection
}

Menopausal symptoms: Women

Mental confusion (delirium)

Mouth sores (mucositis)

Nausea and vomiting

Nervous system disturbances

Neutropenia

Pain

Sexual disorders

Shortness of breath (dyspnea)

Skin problems

Sleeping problems (insomnia)

Sleeping problems: Hypersomnia, somnolence syndrome, and nightmares

Superior vena cava syndrome

Taste changes

Thrombocytopenia

Tiredness (fatigue)

Weight gain

Weight loss 